\title{
SEMISIMPLE REPRESENTATIONS AND AFFINE RINGS
}

\author{
DANIEL R. FARKAS
}

\begin{abstract}
If all finite-dimensional representations of an affine algebra are
\end{abstract} semisimple, then there are only finitely many representations of each degree.

In their monograph [1] on the representations of infinite groups, Lubotzky and Magid refer to a theorem of Weil as a "pure representation-theoretic statement. However, the only proofs we know of this use cohomology and the geometry of the varieties of representations." The result in question states that if $\Gamma$ is a finitely generated group and every finite-dimensional representation is semisimple, then there are only finitely many isomorphism classes of $\Gamma$-representations for each degree.

The purpose of this short note is to present another proof of that theorem, which is an application of the basic propositions from the theory of polynomial identity rings.

In what follows, $k$ is a field. Our goal is to prove the following

THEOREM. Let $R$ be a finitely generated $k$-algebra. If every $R$-module finite dimensional over $k$ is semisimple, then there are only finitely many $n$-dimensional modules (up to homomorphism) for each natural number $n$.

We begin with some obvious reductions. Any $n$-dimensional $R$-module gives rise to a representation of $R$ as $n \times n$ matrices over $k$. Thus an $m$-dimensional representation with $m \leq n$ factors through the ideal generated by all evaluations of the standard multilinear polynomial in $2 n$ variables. In other words, to prove that there are only finitely many $n$-dimensional modules, it suffices to assume that $R$ satisfies a polynomial identity. Moreover, since we assume that all finite-dimensional modules are semisimple, they are all annihilated by the Jacobson radical. So, at the least, we may add the assumption that $R$ is semiprime. We replace the original theorem with a new one.

THEOREM. Let $R$ be a finitely generated, semiprime p.i. algebra over $k$. If every finite-dimensional $R$-module is semisimple, then $R$ is finite dimensional.

ProOF. As a consequence of Procesi's Nullstellensatz [2,V.2], $R$ satisfies the ascending chain condition on prime ideals and the zero ideal is the intersection of finitely many prime ideals. Hence any infinite-dimensional counterexample to the theorem can be assumed to be an affine prime ring with classical Krull dimension 1. (The reader should notice that Wedderburn's Theorem is being used: a finitedimensional prime ring is simple.) A well-known application of the Artin-Tate

Received by the editors August 1, 1986.

1980 Mathematics Subject Classification (1985 Revision). Primary 20E35, 20H05, 16A38.

Key words and phrases. Finite-dimensional representations, polynomial identities.

The author was partially supported by the National Science Foundation. 
Lemma and central polynomials [4] says that such a ring is a finitely generated module over its center, which is affine as well. In particular, $R$ is left noetherian.

Let $P$ be a maximal two-sided ideal of $R$. By Kaplansky's Theorem, $R / P$ is a finite-dimensional central simple algebra over its center $Z$; by the Artin-Tate Lemma $Z$ is a finitely generated $k$-algebra. Hilbert's Nullstellensatz now implies that $R / P$ is a finite-dimensional simple $k$-algebra. Consider $P / P^{2}$ as an $R / P$ module. Since $R$ is noetherian, $P$ is finitely generated. Therefore both $P / P^{2}$ and $R / P$ are finite dimensional over $k$. The semisimplicity hypothesis of the theorem allows us to conclude that $R / P^{2} \simeq R / P \oplus P / P^{2}$ as $R$-modules. But then $P$ annihilates $R / P^{2}$. That is, $P=P^{2}$.

We can choose $P$ more carefully. By the Nullstellensatz, $R$ is semiprimitive. Consequently, it is a subdirect product of matrix rings over finite extension fields of $k$. This means we can find a maximal ideal $P$ so that the p.i. degrees of $R$ and $R / P$ coincide. Then $R$ localized at the complement $S$ of $P \cap Z$ in $Z$ is a local noetherian ring with unique maximal ideal $P_{S}[4 ; 3,1.7]$. Now $P_{S}=P_{S}^{2}$, so Nakayama's Lemma yields $P_{S}=0$. Since $R$ is prime, we obtain $P=0$.

\section{REFERENCES}

1. A. Lubotzky and A. R. Magid, Varieties of representations of finitely generated groups, Mem. Amer. Math. Soc., No. 336 (1985).

2. C. Procesi, Rings with polynomial identities, Dekker, New York, 1973.

3. L. H. Rowen, Polynomial identities in ring theory, Academic Press, New York, 1980.

4. L. W. Small, Rings satisfying a polynomial identity, Vorlesungen, Univ. Essen, Heft 5, 1980.

Department of Mathematics, Virginia Polytechnic Institute and State UNIVERSITY, BLACKSBURG, VIRGINIA 24061 